\title{
Toward an Exosome-Based Therapeutic Strategy in Regenerative Dentistry
}

\author{
Yeon Hee Lee*1, Il Keun Kwon², Jae Min Cha ${ }^{3}$ \\ ${ }^{1}$ Department of Orofacial Pain and Oral Medicine, Kyung Hee University, Republic of Korea \\ ${ }^{2}$ Department of Dental Materials, School of Dentistry, Kyung Hee University, Republic of Korea \\ ${ }^{3} 3$ D-Stem Cell Engineering Laboratory, Department of Mechatronics, College of Engineering, Incheon National University, Republic of Korea
}

Received: 制: December 05, 2018; Published: 制: December 12, 2018

*Corresponding author: Yeon Hee Lee, Clinical Assistant Professor, Department of Orofacial Pain and Oral Medicine, \#613 Hoegidong, Dongdaemun-gu, Seoul, 02447, Republic of Korea

\begin{abstract}
Exosomes are small extracellular vesicles of endosomal origin and have been receiving much attention in the dental field. Their role was considered to be only as a waste disposal system, but now they are emerging as a class of signal mediators. Significantly, their biochemical content includes not only lipids and proteins, but also nucleic acids, mainly miRNAs and mRNAs. They are being considered as attractive vehicles for cargo delivery to target cells. Exosomes also have regenerative and immunomodulatory properties, characteristics that are encouraging their application for regenerative dentistry. Considering the potential role of exosomes in tissue regeneration and their advantages over cell-based therapy, their applications in the dental field will continue to be studied.
\end{abstract}

Keywords: Exosome; Extracellular vesicle; Mesenchymal stem cell; Regeneration; Dentistry

Abbreviations: miRNAs: Micro RNAs; mRNAs: Messenger RNAs; MSC: Mesenchymal Stem Cell

\section{Introduction}

Exosomes are a type of extracellular vesicles that are secreted via exocytosis from late endosome multivesicular bodies [1] (Figure 1A). Exosomes are nano-vesicles measuring 40 to $150 \mathrm{~nm}$, first discovered in the supernatant of cultured sheep erythrocytes in 1983 [2]. Since then, the progress of bioscience and technology has revealed that their role is not limited to be a waste disposal system; they are now emerging as a class of signal mediators with cellular component release [3-5]. They also have regenerative and immunomodulatory properties, characteristics that are encouraging their application for therapeutic purposes [4,5]. Although exosomal functions have already been widely explored in regenerative medicine, the potential for regulating tissue repair and regeneration in the dental field has not drawn nearly as much attention [6]. Therefore, the use of exosomes for human cleft lip and palate reconstruction, periodontal regeneration, osteoinductive roles in orthodontic treatment, healing in facial bone fracture, and chondral and muscular regeneration in temporomandibular disorders will continue to be studied.

\section{Exosome-Based Therapeutics in Regenerative Dentistry}

Exosomes are increasingly gaining the attention of the scientific community because of their small size and ubiquitous presence in almost every fluid of the human body, such as saliva, urine, plasma, synovial fluid, breast milk, amniotic liquid, seminal fluid, ascites, and cerebrospinal fluid [5]. Exosomes are enveloped by a lipid bilayer enriched in cholesterol, ceramide, and sphingomyelin. The membrane of exosomes is also abundant in some tetraspanins such as CD9, CD63, and CD81, that could be used as markers for identifying exosomes. The internal contents of exosomes are enriched in special biomolecules, functional proteins, and nucleic acids, including microRNAs (miRNAs), messenger RNAs (mRNAs), and even DNA (Figure 1B) [3,7]. With these components, exosomes have been identified as another vital mediator of paracrine communication [8]. Paracrine signaling is of major importance in maintaining cellular homeostasis, and it also plays a key role in the onset and development of many diseases [9]. 


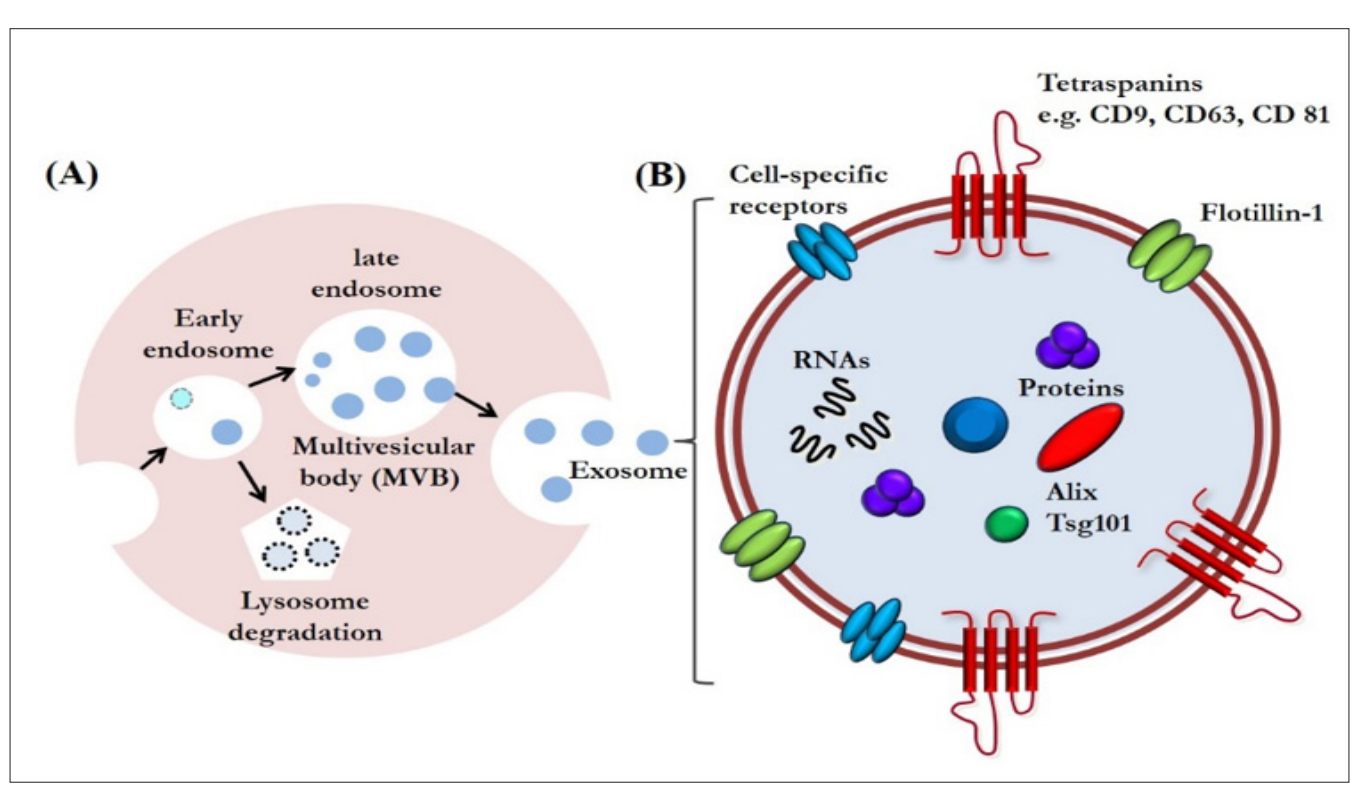

Figure 1:

A. Exosome biogenesis. Exosomes are an end-product of the endocytic recycling pathway. First, endocytic vesicles are formed at the plasma membrane and fuse to form early endosomes. These mature and become late endosomes. After further processing, exosomes are released through membrane fusion.

B. An enlarged exosome showing a variety of common exosomal surface markers (e.g., tetraspanins such as CD9, CD63, and CD81 and lipid raft-associated proteins including flotillin-1), as well as internal markers such as Alix and Tsg101. Each exosome also contains and transfers small RNAs and other cytoplasmic proteins and cell-specific receptors, which can be transferred to recipient cells.

Furthermore, their relative stability in circulating body fluids and their ability to pass biological barriers have prompted investigations aimed at using them for therapeutic purposes [10]. Finally, a large collection of evidence shows that exosomes are important regulators of many biological functions, such as tissue regeneration, immune response, and tissue homeostasis [11]. With these properties, they would be useful for innovative approaches in tissue regeneration against bone destruction or defect in the dental field. We could reasonably visualize a cell-free therapy using exosomes for tissue regeneration. It is worthy to be mentioned that mesenchymal stem cell (MSC)-derived exosomes might constitute a compelling alternative because of their advantages over the corresponding MSCs: they are less complex and even smaller than cells, so they are easier to produce and store, and have the potential to avoid some of the regulatory or legal issues that MSCs face [12].

It also would avoid the risks related with direct stem cell transplantation, such as hyper-immune reaction or immune rejection, teratoma formation, and the reduced regenerative capacity of engrafted cells [13]. Therefore, MSC-derived exosomes may be an ideal therapeutic tool for regenerative dentistry in near future.

In addition, MSC-derived exosomes are particularly promising candidates for developing cell-free therapy in several fundamental biological processes, such as recruitment of inflammatory cells, neovascularization, and coagulation [14]. Thus, they have vital importance in ensuring the appropriate inflammatory reaction after injury, which would improve tissue repair and regeneration. Angiogenesis is of vital importance in various physiological processes including cutaneous wound healing and/or tissue regeneration. Exosomes released by human adipose-derived MSCs can significantly promote endothelial cell angiogenesis in vitro and in vivo [15-17].

Furthermore, exosomes derived from human amniotic epithelial stem cells have the regenerative potential to heal full-thickness skin defect in rats [18]. Exosomes derived from human MSCs have therapeutic effects on osteochondral defect and eventually lead to cartilage repair [19]. However, the precise underlying molecular mechanisms of these beneficial effects have not yet been determined, which may be a highly orchestrated physiological process consisting of a complex event. Recent evidence suggests that exosomes secreted by most cell types can mediate transfer of their cargo. Theoretically, endogenous exosomes could be reasonable candidates for natural drug delivery because of their small size, permeability of physiological barriers, nontoxicity, low immunogenicity, and stability in circulation [20]. Emerging exosomal engineering strategies have laid the foundation for achieving this goal [21]. In the near future, with assistance of exosomes engineered with anti-inflammatory drugs or compounds, clinicians might be able to modulate the inflammatory response soon after tissue damage occurs.

In addition, modifying the surface of exosomes by adding proteins with affinity to injured cells and tissues could precisely 
drive exosomes to a target site [22]. Further, with encapsulation of nucleic acid into exosomes, exosomes could carry a miraculous therapeutic potential for tissue regeneration through modulating the microenvironment of their target cells [21]. Despite extensive evidence, the potential roles of exosomes in tissue repair and regeneration have not been fully elucidated. The certain contents and properties of exosomes that are capable of promoting tissue regeneration are still unclear. It would be also of great significance in identifying the variations in exosomal amounts following injury, because excessive exosome recruitment can lead to further tissue damage by persisting the inflammatory response. Furthermore, great efforts still needed for developing optimized methods for exosomal isolation and purification.

\section{Conclusion}

Although there is much that remains to be investigated in the field of exosomal research, the unique properties of exosomes clearly represent new therapeutic strategies for tissue repair and regeneration in the dental field. It is reasonable to believe that more regenerative potential of exosomes will be discovered in the future. The innovative strategy of using exosomes is obviously suggesting new options for regenerative medicine, although there are areas that need further research before clinical application. Using the exosome-based therapeutic strategies, we might be able to reduce safety concern and immunogenicity problems in regenerative dentistry.

\section{Financial support}

This research was supported by the Bio \& Medical Technology Development Program of the National Research Foundation (NRF) funded by the Korean government (MSIT) (No. 2017M3A9E4048170).

\section{Conflict of interests}

The authors have read the journal's policy on conflicts of interest and authorship agreement, and we have disclosed nonpotential conflicts of interest.

\section{Acknowledgement}

\section{None.}

\section{References}

1. Ferguson SW, Nguyen J (2016) Exosomes as therapeutics: The implications of molecular composition and exosomal heterogeneity. Journal of controlled release: official journal of the Controlled Release Society 228: 179-190.

2. Pan BT, Johnstone RM (1983) Fate of the transferrin receptor during maturation of sheep reticulocytes in vitro: selective externalization of the receptor. Cell 33(3): 967-978.

3. Khalyfa A, Gozal D (2014) Exosomal miRNAs as potential biomarkers of cardiovascular risk in children. Journal of translational medicine 12 : 162-162.

4. Urbanelli L, Buratta S, Sagini K, Ferrara G, Lanni M, et al. (2015) Exosomebased strategies for Diagnosis and Therapy. Recent patents on CNS drug discovery 10(1): 10-27.
5. Urbanelli L, Magini A, Buratta S, Brozzi A, Sagini K, et al. (2013) Signaling pathways in exosomes biogenesis, secretion and fate. Genes 4(2): 152170 .

6. Stanko P, Altanerova U, Jakubechova J, Repiska V, Altaner C (2018) Dental Mesenchymal Stem/Stromal Cells and Their Exosomes. Stem cells international pp. 8973613-8973613.

7. Camussi G, Deregibus MC, Bruno S, Cantaluppi V, Biancone L (2010) Exosomes/microvesicles as a mechanism of cell-to-cell communication. Kidney international 78(9): 838-848.

8. Gangoda L, Boukouris S, Liem M, Kalra H, Mathivanan S (2015) Extracellular vesicles including exosomes are mediators of signal transduction: are they protective or pathogenic? Proteomics 15(2-3): 260-271.

9. Muller E, Wang W, Qiao W, Bornhauser M, Zandstra PW, et al. (2016) Distinguishing autocrine and paracrine signals in hematopoietic stem cell culture using a biofunctional microcavity platform. Scientific reports 6: 31951 .

10. Saraiva C, Praca C, Ferreira R, Santos T, Ferreira L, et al. (2016) Nanoparticle-mediated brain drug delivery: Overcoming blood-brain barrier to treat neurodegenerative diseases. Journal of controlled release: official journal of the Controlled Release Society 235: 34-47.

11. Robbins PD, Morelli AE (2014) Regulation of immune responses by extracellular vesicles. Nature reviews Immunology 14(3): 195-208.

12. Katsuda T, Kosaka N, Takeshita F, Ochiya T (2013) The therapeutic potential of mesenchymal stem cell-derived extracellular vesicles. Proteomics 13(10-11): 1637-1653.

13. Herberts CA, Kwa MSG, Hermsen HPH (2011) Risk factors in the development of stem cell therapy. Journal of translational medicine 9: 29-29.

14. Lou G, Chen Z, Zheng M, Liu Y (2017) Mesenchymal stem cell-derived exosomes as a new therapeutic strategy for liver diseases. Experimental \& molecular medicine 49(6): e346-e346.

15. Behera J, Tyagi N (2018) Exosomes: mediators of bone diseases, protection, and therapeutics potential. Oncoscience 5(5-6): 181-195.

16. Liang X, Zhang L, Wang S, Han Q Zhao RC (2016) Exosomes secreted by mesenchymal stem cells promote endothelial cell angiogenesis by transferring miR-125a. Journal of cell science 129(11): 2182-2189.

17. Hu L, Wang J, Zhou X, Xiong Z, Zhao J, et al. (2016) Exosomes derived from human adipose mensenchymal stem cells accelerates cutaneous wound healing via optimizing the characteristics of fibroblasts. Scientific reports 6: 32993-32993.

18. Zhao B, Zhang Y, Han S, Zhang W, Zhou Q et al. (2017) Exosomes derived from human amniotic epithelial cells accelerate wound healing and inhibit scar formation. Journal of molecular histology 48(2): 121-132.

19. Wang Y, Yu D, Liu Z, Zhou F, Dai J, et al. (2017) Exosomes from embryonic mesenchymal stem cells alleviate osteoarthritis through balancing synthesis and degradation of cartilage extracellular matrix. Stem cell research \& therapy 8(1): 189-189.

20. Ohno S, Kuroda M (2016) Exosome-Mediated Targeted Delivery of miRNAs. Methods in molecular biology (Clifton, NJ) 1448: 261-270.

21. Gilligan KE, Dwyer RM (2017) Engineering Exosomes for Cancer Therapy. International journal of molecular sciences 18(6).

22. Tamura R, Uemoto S, Tabata Y (2017) Augmented liver targeting of exosomes by surface modification with cationized pullulan. Acta biomaterialia 57: 274-284. 
ISSN: 2574-1241

DOI: 10.26717/BJSTR.2018.12.002190

Yeon Hee Lee. Biomed J Sci \& Tech Res

(c) (P) This work is licensed under Creative

Submission Link: https://biomedres.us/submit-manuscript.php

$\begin{array}{ll}\text { BIOMEDICAL } & \text { Assets of Publishing with us } \\ \text { RESEARCHES } & \text { - Global archiving of articles } \\ \text { - Immediate, unrestricted online access }\end{array}$

